\title{
VUE DES ETATS-UNIS
}

\section{AMBIGUITE DES PROCES STALINIENS:}

\section{INTERPRETATION DE LA PENSEE DE MERLEAU-PONTY}

Depuis l'accession de Gorbachev au pouvoir, les "Révolutions" de 1989 et les transformations internes en U.R.S.S., beaucoup a eté ecrit sur l'avenir du communisme, ou plus précisément sur son échec. Jamais n'a-t-il été plus évident de révaluer Humanisme et terreur (1947) de Maurice Merleau-Ponty quant à son propre mérite, et non pas par rapport à son autre critique du communisme, Les Aventures de la dialectique (1955). Depuis quelques années la philosophie de Merleau-Ponty est jugé fondamentalement sur son échec à reconnaître l'inhumanite marxiste montrée par l'U.R.S.S. dans les années trente. Sa philosophie n'a pas été "à la mode" depuis l'éclipse de l'existentialisme et l'avènement d'autres philosophes comme Foucault, Derrida, et Deleuze. Or avec cette "Révolution" dans le cadre communiste et la "démocratisation" des pays dans l'ombre de l'U.R.S.S., la vérité que Marx a donnée à la pensée philosophique et politique sur la condition humaine peut être regardée telle que Merleau-Ponty l'a décrite il y a presque un demi-siècle, même si on considère que cette expérience est moins un refus du marxisme qu'un rejet du stalinisme.

Pour Merleau-Ponty et sa génération, le mouvement le plus puissant qui les a marqués profondément fut la guerre. Dans l'article, "La Guerre a eu lieu," Merleau-Ponty écrit très franchement que ses concitoyens furent surpris de cet événement cataclysmique. Dans un sens, 
l'époque de l'avant-guerre fut un "jardin terrestre", "... un certain licu de paix, d'expérience exceptionnelle, et nous ne savions pas que ce fût là un sol à defendre, nous pensions que cétait le lot naturel des hommes" (SNS, 246). La guerre changea tout. Avec la défaite de la France en 1940, il n'y avait plus d'innocents; le jardin fut perdu, et la conduite de personne n'était irréprochable. Soit si l'on restait en France, soit si on la quittait, "personne n'a les mains propres" (SNS, 259).

La guerre plus que rien d'autre lui a appris l'histoire et lui a permis de reconnaître que la condition quotidienne---la liberté réelle-était pleine de sens, surtout là où elle touche à l'inhumanité de l'homme envers d'autres hommes. La guerre a surtout créé une division entre le temps antérieur et ultérieur et cette division a provoqué un changement radical dans la vie des hommes. La découverte qu'un homme peut traiter autrui non pas en tant qu"homme", "Français", "Allemand", ou "prolétaire" les a touchés, à leurs racines, c'est-à-dire dans leur humanite. Tous les existentialistes éprouvaient cette radicalité envers leurs origines comme être humain et cette radicalité était étayée de leurs expériences. ${ }^{1}$

La Résistance fut le jalon sur lequel les philosophes de la génération de Merleau-Ponty se sont appuyes pour faire référence à un événement courant dans l'histoire française: la Révolution. La situation de la politique française après la guerre a exigé qu'une question précise sur le problème du communisme soit soulevée. Il fallait définir la position de l'U.R.S.S. vis-à-vis de l'Occident et réduire les différends qui allaient détériorer les relations entre l'U.R.S.S. et les Etats-Unis.

Merleau-Ponty avait dejà décrit sa position en 1945 dans "Pour la vérite" (SNS, 271-303). Selon lui certains thèmes dans les écrits marxistes furent bouleversés. La dialectique, considétée par Marx comme le thème le plus important dans ses ecrits, fut "déraillée" (SNS, 228-229). L'histoire passe selon son mode d'emploi et de production dans la transformation de la nature de son travail. Cette action devient dialectique du fait de la participation humaine. La classe ouvrière, en

\footnotetext{
${ }^{1}$ Rudolph M. Meyer. "Maurice Merleau-Ponty und des Schicksal des Franzosischen Existentialismus." Philosophische Rundschau 5 Heft 3/4 (1955): 131.
} 
lutte avec les autres classes, incarne la dialectique entre l'Histoire et l'Homme. Chaque époque révèle la lutte entre ceux qui détiennent le pouvoir et ceux qui le veulent. La bourgeoisie, selon Marx, est la classe dominante depuis l'ascension du capitalisme et les forces économiques indiquent que la chute de cette classe est imminente. La prise du pouvoir de la classe ouvrière, le prolétariat, dans l'U.R.S.S., en 1917, fut la culmination de toute la pensée marxiste et la révolution soviétique est devenue le point de référence à tout mouvement prolétarien futur.

En 1945, la situation a changé. Les procès des années trente, menés par Staline, avaient un double but: éliminer ses ennemis et attaquer les vieux bolcheviks. Cette sorte d'action renverse la logique interne du communisme. Le Parti, fondé par Lénine pour guider le prolétariat, est selon lui la conscience de cette classe (HT, 106). Si le prolétariat cède au Parti ses pouvoirs pour le laisser exercer sa dictature--la Dictature du proletariat---toute violence contre les ennemis de cette classe est justifiée parce que "le triomphe de la vérité du prolétariat"(HT, 106) est la règle absolue de toute action marxiste. Le secret du marxisme et celui qui lui donne sa puissance est la façon avec laquelle le marxisme utilise la violence contre ses ennemis. La faiblesse du liberalisme occidental est de nier que la violence peut être exercée contre ses ennemiṣ et qu'elle est exercée seulement contre ses actions. L'hypocrisie du libéralisme consiste en l'ídée que la violence n'occupe aucune place dans une civilisation alors qu'elle l' utilise contre ses propres citoyens. On ne peut pas utiliser la violence pure. Seulement si "cette violence a un sens, s'il est possible de la comprendre, d'y lire un développement rationnel, d'en tirer un avenir humain" $(\mathrm{HT}, 116)$ peut-on l'accepter comme valable. Cet avenir humain fut l'espoir de la Révolution de 1917.La violence fut acceptée comme une condition fonda-mentale de l'homme par Marx et Hegel car la lutte est la condition de l'existence humaine. Le premier acte que l'homme fait pour devenir humain est un acte de violence contre Autrui. Cet acte, conduit par le désir de détruire l'Autre, rend l'homme capable d'exister avec Autrui dans un monde commun. ${ }^{2}$ Cette lutte ne cesse jamais et, à partir de ce premier acte, à la fois vraiment violent et

${ }^{2}$ Hegel. La Phenoménologie de l'esprit. Traduction de Jean Hyppolite. Tome I. (Paris: Aubier, 1939): 159. 
humain, commence la vie humaine. L'inégalité constante est en réalité la raison de l'existence malheureuse de l'homme: une lutte qui le suit partout dans sa présence au monde; ces deux conditions de son existence seraient résolues par le prolétariat et sa mission historique. Voila le principe fondamental du marxisme.

Cette classe qui va résoudre l'injustice du monde exige que l'individu supprime son individualité ou sa liberté formelle pour atteindre l'universalité dans la classe prolétarienne. ${ }^{3}$ Cette suppression, pourtant violente, est preférée à la violence quotidienne du capitalisme où les principes de l'égalité, de la liberté et de la fraternité sont inscrits dans les les lois, mais, en fait, dans la vie quotidienne, l'utilisation de cette violence se tourne contre les citoyens qui ne réalisent jamais ces principes dans leur vie formelle. Par la suppression de son identité comme individu, aliéné par la séparation qui existe entre lui et son objet de travail, l'homme réalise en lui-même, concrètement, une liberté réelle qui, tout a coup, lui permet de surmonter--même "sursumer"--son aliénation et son esclavage et il se soumet à une universalité concrète qu'il organise et dirige vers un monde et vers une existence humaine. Cette nouvelle humanité, née de la violence prolétarienne qui n'est ni une moralité chrétienne, c'est-à-dire une fausse moralité qui s'occupe de ce qu'on pense, ni une moralité kantienne qui s'occupe de ce qu'on veut. La vraie moralité intersubjective n'est rien d'autre que la moralité prolétarienne, portée sur les actions des hommes et des résultats de leurs actions (HT, 123). La violence prolétarienne supprime l'individualité et la liberté quotidienne pour atteindre la vraie liberté, c'est-à-dire, la liberté dans l'avenir qui donnera à l'homme une existence et un monde humain. Donc, le problème que confronte le marxisme est un problème de l'inter-subjectivite, un problème éthique. Si l'on a déjà accepté que la violence est nécessaire et même que l'humanité commence à partir de la violence, la vio-

\footnotetext{
${ }^{3} \mathrm{La}$ distinction entre les termes allemands d"'Abschaffung" et d' "Authebung" est très importante dans la pensée marxiste, et cette distinction est perdue dans la traduction anglaise et française. Le traducteur de la Science de la logique a essayé de résoudre ce problème par le néologisme "sursumer," in Science de la logique. Traduction par PierreJean Labarrière et Gwendoline Jarczyk (Paris: Aubier-Montaigne, 1972), Note infrapaginale, 38 .
} 
lence elle-même, n'est pas une question essenticlle pour le marxiste. Le problème se pose ainsi: comment peut-on utiliser la violence, qui est vraiment inhumaine, pour améliorer la condition de l'homme? La solution à l'injustice dont le prolétariat est la clef, commence par le renversement du problème du maître et de l'esclave. La mission historique du prolétariat est de reconnaître l'homme comme homme, non pas comme esclave ou maître. Il n'y a ni maîtres ni esclaves, mais seulement I homme reconnudans une sociêté, sans classe certes, mais réellement humaine. Donc, la violence prolétarienne n'a pas de sens hors d'une référence au prolétariat: "La violence, la ruse, la terreur, le compromis, enfin la subjectivité des chefs et du Parti... trouvent leur limite et leur justification en ceci qu'ils sont au service d'une société humaine" (HT, 129).

L'emploi du pouvoir au service du prolétariat distingue l'état bolchevique de l'état fasciste. Du point de vue du totalitarisme les deux systèmes de gouvernement sont égaux, car chacun utilise l'individu pour le service de l'Etat qui est suprême dans son pouvoir. Or, quand on analyse le contenu des deux formes de gouvernement, ce qui rend l'etat bolchevique "humain" est précisément la théorie du prolétariat. Le fascisme est "une mimique du bolchevisme. Parti unique, propagande, justice d'Etat, vérité d'Etat, le fascisme retient tout du bolchevisme, sauf l'essentiel, c'est-à-dire la théorie du prolétariat" $(\mathrm{HT}, 139)$.

Cette theorie est vraiment le noyau de la doctrine marxiste. Il faut noter que Marx est ambigu dans ses écrits en ce qui concerne le rôle du Parti à l'égard du prolétariat, mais que Lénine assigne à ce dernier un rôle plus important et plus dominant. Selon sa théorie de la spontanéité des classes, le prolétariat ne peut pas éveiller lui-même une consciencede-soi et donc renverser sa domination par la bourgeoisie, mais il prend la position que le prolétariat ne peut pas exister sans lui. Le prolétariat n'est pas capable de se gouverner. D $\ell_{j} \mathrm{j}$ dans cette transformation du rôle du Parti selon Lénine sont cachées les graines du pouvoir absolu, c'est-à-dire du totalitarisme. Le prolétariat n'est plus essentiel au mouvement révolutionnaire. L'effort du Parti, ses chefs et la conscience des chefs, tous trois joueront dans le système du pouvoir, dans le système bureaucratique, et prendront une position "en avant" du prolétariat. Déja Merleau-Ponty a vu que "le mouvement prolétarien lui-même a cessé 
d'être le terme de référence de la pensêe communiste" (HT, 154). Il l'appelle "la nouvelle politique" et en prenant le terme "néo-communisme" (HT, 158), Merleau-Ponty se demande si cette révision du léninisme peut être inevitable et surtout si cette nouvelle politique "ne peut, comme l'ancienne, concorder avec le voeu de la conscience" $(\mathrm{HT}, 155)$. Avec ce changement de l'énergie du prolétariat par les chefs, le marxisme, au sens classique, n'existe plus en termes politiques. La dialectique est brisée en tant que but de l'esprit révolutionnaire et politique prolétarienne (HT, 154). Les procès de Staline, loin d'être étranger à la mentalité soviétique ou communiste, sont le résultat d'une logique interne qui réalise la dialectique historique et qui la bouleverse à la fois.

Ce bouleversement de la dialectique historique, et "la fin" de l'histoire au sens prolétaire, s'est réalisé dans les procès eux-mêmes ainsi que dans la "Grande Terreur" stalinienne. Selon Marx, la violence ne peut être utilisée que dans le but d'établir le pouvoir prolétarien. Toute déviation de ce but produit un "déraillement de l'Histoire" (SNS 228-229). Ce "déraillement" se trouve justement dans les procès de Staline. Même si l'on se demande s'ils se sont vraiment faits dans le mouvement de l'Histoire, il faut au moins admettre que "la direction stalinienne procède par une série de zigzags et non pas selon une ligne vraiment marxisten (HT, 98). Les jugements de Moscou sur les procès peuvent être reconnus comme étant différents ou séparés de ceux de l'Histoire, soit comme un développement stalinien du marxisme-léninisme. Une raison et une seule peut justifier les procès: celle de l'affaiblissement de l'U.R.S.S. Il faut se rappeler que la violence a besoin de l'idéologie pour la justifier et, aussi longtemps que l'idéologie justifie la violence, c'est-à-dire la violence prolétarienne, on peut dire qu'elle est "morale". Quand la violence est vidée de l'idéologie, il est plus difficile de la considérer comme "morale." Or plus la violence s'accroît, plus il faut avoir une idéologie pour la justifier. ${ }^{4}$ Le paradoxe des procès consiste à les mettre dans la ligne révolutionnaire où ils ne se justifient que par leur contact avec l'Histoire. C'est aussi un fait que ces procès condamnaient les vieux révolutionnaires bolcheviks qui avaient participé à la Révolution. Ils ne

"Hanna Arendt. On Violence. (New York: Harcourt, Brace, Jovanovich, 1970): 77. 
révolutionnaires bolcheviks qui avaient participé à la Révolution. Ils ne sont considérés comme traîtres que si l'on voit "dans la situation présente [que] l'opposition a fait le jeu de l'ennemi de classe" (HT, 107).

Les procès servaient, par conséquent, deux voies de pensée d'après l'analyse de Merleau-Ponty. Premièrement, dans le mouvement révolutionnaire dialectique, la violence que les procès ont infligée, peut être justifiée si on les considere comme phase logique de ce mouvement. Cela veut dire qu'ils se font a l'intérieur du concept historique qui rend toute analyse de l'exterieur superflue et non valable parce qu'il n'y a pas de référence a la moralité de la fin et des moyens hors du mouvement même. "Le propre du marxisme est de ne pas distinguer entre la fin et les moyens, et il n'y a pas en principe de politique moins hypocrite et moins machiavélique" (SNS, 264). Toute critique des proces est vue d'une perspective hors du milieu où ils ont eu lieu. Pour cette raison, les procès se justifient aux yeux de l'Histoire. Deuxièmement, fondés sur la première raison ci-dessus, les procès ont bouleversé la dialectique du mouvement révolutionnaire justement par leur conclusion qui oriente la violence contre ceux qui composent la classe ouvrière.

En ce qui concerne la théorie et la praxis, on peut constater qu'au moment où les procès détruisent la Révolution en la réalisant, la théorie marxiste est annulée pour bien concrétiser son côté de praxis. En tant que praxis, le "néo-communisme", qui existe après la séparation de la théorie et de la praxis, dépend d'avantage d'un pragmatisme du pouvoir que d'un essor de l'intérêt prolétarien. Cette fonction a, au fond, une tendance vers un totalitarisme nu. L'entrelacement intime que Montaigne imagina de la théorie et de la praxis---c'est-à-dire le sens que l'une peut avoir pour l'autre et inversement---s'applique sans difficulté à l'expression d'un faux marxisme où "toute doctrine, séparée de ce que nous faisons, menace d'être menteuse..." $(\mathrm{S}, 252)$. Ceux qui interprètent le marxisme du point de vue "orthodoxe" ne font qu'une analyse où "nous ne trouvons dans les textes que ce que nous y avons mis..."(PP, ii) et réduisent le marxisme à une vérité historique, à un instrument de propagande pareil aux vérités qui n'ont pas duré dans l'histoire.

Si l'interpétation ci-dessus est valable, les procès se sont justifiés 
du point de vue marxiste même si l'on n'accepte pas les suppléments que Staline y a ajouté. Selon la logique de la dialectique, il faut protéger la Révolution et, par conséquent, le pays où elle s'est établie. Or, sans l'essentiel révolutionnaire, c'est-à-dire le prolétariat, la Grande Terreur et les proces n'ont aucun sens. Il n'y a pas de différence entre les événements qui s'y sont déroulés et ceux du troisième Reich. La séparation qui s'est effectuee entre la théorie et la praxis vise un autre probleme chez Merleau-Ponty et s'avère être une menace pour son point de départ: le mélange du marxisme et de l'existentialisme. Ce n'est pas ici le moment opportun pour poser une si grande question afin de résoudre ce probleme, car un "Qu'est-ce que l'existentialisme?" serait trop général. L'existentialisme de Merleau-Ponty n'est pas "pur," car il a emprunté des éléments à la phénoménologie de Husserl aussi bien qu'à la philosophie de Heidegger pour creer sa propre definition.

Au fond, l'existentialisme de Merleau-Ponty commence avec l'expérience du corps comme contact primordial avec le monde et on ne peut pas séparer le corps de la conscience du corps. Conscience et corps, dans l'expérience, ne peuvent pas être séparés parce que ni dans l'expérience de soi ni dans l'expérience de l'autre ne vise-t-on les deux sépares l'un de l'autre, dans un contexte humain. "L'amour n'est pas du corps seulement puisqu'il vise quelqu'un, et il n'est pas de l'esprit seulement puisqu'il vise dans son corps" $(\mathrm{S}, 254)$. Comme Descartes a essayé de le démontrer, on ne peut concevoir qu'ensemble l'essence et l'existence de Dieu.

Donc, on n'a pas besoin de chercher l'origine de l'homme car son origine se trouve dans l'action. L'expérience du monde comme un "dejà la" ne nécessite pas non plus de chercher son origine. Il ne reste que de s'y engager. Ainsi se dessinent deux pôles dans la pensée de MerleauPonty: l'un qui com-prend le sujet, individuel et unique, même "historique $^{n}$, et l'autre qui comprend l'objet, le monde qui est l'espace humain des activités. L'essence de l'activité au monde est celle de l'individu engage face aux autres dans un rapport qui transforme les problemes individuels en problème d'intersubjectivite. L'homme individuels'engage avec l'homme social et ce contact incarne vraiment une dialectique irréductible. L'action individuelle n'est pas supprimée par son contact 
avec le groupe, et la tension entre l'individu et le groupe n'est jamais résolue..$^{5}$

Ce n'est pas du tout le cas dans le marxisme. Chez Marx, il n'est possible de voir l'essence humaine presente dans l'homme individuel qu'avant Le Manifeste communiste. L'individu, pour tranformer (sursumer) son individualité et son aliénation vis-à-vis de son objet de travail, doit supprimer sa propre individualité dans la classe ouvrière. Or, dans les Thèses sur Feuerbach, Marx a écrit sans ambiguitté qu'il n'y a pas d'essence humaine hors de la société. "L'essence humaine n'est pas une abstraction inhérente à l'individu isolé. Dans sa réalité, elle est l'ensemble des rapports sociaux".6 L'homme individuel est "une mystification" et n'a pas de valeur dans le système de Marx. On voit ici dans la VIème Thèse la base de la transformation du marxisme chez Lénine où il établit le Parti pour diriger le proletariat et, si les individus ne le suivent pas, cela n'a aucune importance car au moment où le Parti le dirige, les individus se sont deja soumis a la classe ouvrière. Une fois soumise au proletariat, ce n'est pas l'individu qui contribue à la dialectique, mais le proletariat comme groupe. La violence faite par l'individu n' pas de place dans un contexte marxiste que si elle se réfere au but de la lutte des classes: l'établissement du pouvoir prolétarien.

La tension entre l'existentialisme de Merleau-Ponty et sa tendance vers le marxisme est déja evidente dans les dernières pages de la Phénomélogie de la perception (1945). Ayant déjà considéré la pensée objective et l'analyse réflexive dans l'introductionet la première partie de son oeuvre, Merleau-Ponty les applique au problème du prolétariat:

Or, nous avons déjà vu que la pensée cibjective et l'analyse réflexive sont deux aspects de la même erreur, delux manières d'ignorer les phenomènes. La pensée objective déduit la conscience de classe de la condition objective du prolétariat. La réflexion idéaliste réduit la

\footnotetext{
${ }^{5}$ Voir la definition de l'existentialisme dans SNS, 237.
}

${ }^{6}$ Même Thèse sur Feuerbach, cité dans Lucien Sève, Marxisme el théorie de la personnalite. (Paris: Editions sociales, 1972): 88. 
condition prolétarienne a la conscience que le proletaire en prend. La première tire la conscience de classe de la classe definie par des caractères objectifs, la seconde au contraire réduit l"être ouvrier", à la conscience d'Etre ouvrier. Dans les deux cas, on est dans l'abstraction, parce qu'on demeure dans l'alternative de l'en soi et du pour soi. (PP, 506).

On ne définit pas la classe ouvrière comme cela mais d'une manière existentialiste, c'est-à-dire par l'expérience d'être "ouvrier" ou "bourgeois".?

Le moment où Merleau-Ponty distingue le fait d'être ouvrier de celui d'être conscient de ce fait, l'ouvrier a déjà supprimé cette "conscience $\mathrm{de}^{n}$ pour entrer dans la classe ouvriere. Pour Merleau-Ponty il y a dans le choix d'accepter cette "conscience de" l'essentiel de son existentialisme. Or, dans une perspective vraiment marxiste, l'essence humaine ne réside pas dans la conscience d'être ouvrier mais dans l'acte de se soumettre au prolétariat. Le refus de Marx de reconnaître l'individualite comme lieu de l'essence de l'homme s'explique parce que l'homme-individu n'est qu'une abstraction. Au contraire, la route pour atteindre la conscience de classe chez Merleau-Ponty est par expérience de cette ipsiété "ouvrière". Chez Marx le concept de classe est déduit d'une analyse de l'histoire de la lutte des classes et, à travers cette analyse, il semble que Marx tombe dans l'erreur de la pensée objective.

Si cette critique est valable, Merleau-Ponty se sépare de la pensée marxiste de deux façons: premièrement, par sa conception de l'individu et de l'individualite comme la base de la philosophie existentialiste et, deuxièmement, par son aveuglement en ne prenant pas Marx

\footnotetext{
7"Je n'ai pas conscience d'être ouvrier ou bourgeois parce que, en fait, je vends mon travail ou que je suis en fait solidaire de l'appareil capitaliste, et pas davantage je ne deviens ouvrier ou bourgeois le jour où je me décide à voir l'histoire dans la perspective de la lutte des classes: mais 'j'existe ouvrier' ou 'j'existe bourgeois'd'abord, et c'est ce mode de communication avec le monde et la sociêté qui motive a la fois mes projets révolutionnaires ou conservateurs et mes jugements explicites: "je suis un ouvrier" ou "je suis un bourgeois", sans qu'on puisse déduire les premiers des seconds, ni les seconds des premiers. Ce n'est pas l'économie ou la société considérées comme système de forces impersonnelles qui me qualifient comme prolétaire, c'est la société ou l'économie telles que je les porte en moi, telles que je les vis..." (PP, 506).
} 
comme représentatif de la pensée objective. Avec ces contra-dictions a l'intérieur de sa pensée, il ne peut maintenir pour longtemps une position qui joint l'existentialisme au marxisme. Cette sorte d'union aboutira à une rupture interne. Après la mise à jour de PHumanisme et terreur, Merleau-Ponty essaie de montrer qu'une troisième voie est possible ${ }^{8}$. Ni communiste, ni anticommuniste,' il montre, dans ses articles, qu'il considere la guerre-froide comme un cas où l'histoire est depouillé de rationalité par l'aveuglement des acteurs du drame. Il semble, à l'égard de l'U.R.S.S., prendre une position proche à celle de Trotsky un peu avant sa mort car Trotsky lui aussi ressentait l'ambiguïté d'être communiste dans un monde en guerre: la défense de l'U.R.S.S. et en même temps l'augmentation puissante du prolétariat mondial. Pour Trotsky, le stalinisme est contre la Révolution de 1917 et même contre les intérêts du prolétariat qui sont essentiels à l'état soviétique. Toute action contre le stalinisme est action pour l'Etat révolutionnaire soviétique, même si cette action culmine à un démembrement de ce dernier. ${ }^{10}$ La "stalinisation" du monde ne se limite pas aux événements en U.R.S.S., mais elle inclut aussi les actions prises par les pouvoirs et les individus qui étendent son influence. Même l'aide américaine du plan Marshall détermine le durcissement du stali-nisme comme politique sans alternative. Cette aide américaine "à l'Eu-rope est impensable dans les perspectives de l'impérialisme au sens marxiste (comme d'ailleurs dans celle du libéralisme). Elle est dans une très large mesure gratuite" ${ }^{n}{ }^{11}$ Or, si le plan Marshall n'établit pas ouvertement l'impérialisme américain, il

'Pour l'histoire de la troisième voie, voir Michel-Antoine Burnier, Les existentialistes et la politique. (Paris: Gallimard, 1966).

\footnotetext{
9n Nous sommes, nous aussi, contre la morale abstraite. C'est pourquoi nous ne suivons pas les anticommunistes, qui jugent le communisıne sans considérer les problèmes de I'U.R.S.S. Encore faut-il que les valeurs soient reconnaissables sous leur aspect du moment. C'est pourquoi, ne reconnaissant pas dans le communisme aujourd'hui celles de l'humanisme marxiste, nous ne sommes pas communistes" (S, 402).

${ }^{10}$ Voir "La politique paranoiaque," $(S, 324)$.

11"Complexité objective." Les Temps modernes 34 (juillet 1948): 3.
} 
établit au moins Pimperium ${ }^{12}$ et commence une autre sorte de guerre qui est le résultat de la "complexité objective" de la politique Truman. C'est une politique qui retombe dans une sorte de stalinisme ou la paix n'est pas rétablie et le monde se trouve entre deux grandes puissances "encore un peu plus près de la guerre". ${ }^{13}$

De plus en plus dans ses articles, Merleau-Ponty a tendance a s'éloigner de sa position originale exposée dans $\mathbf{P H u m a n i s m e ~ e t ~ t e r r e u r . ~}$ On le remarque surtout dans l'article, "Lecture de Montaigne", où il ecrit que le fameux scepticisme de Montaigne a deux faces: "Il signifie que rien n'est vrai, mais aussi que rien n'est faux. [Montaigne] rejette comme absurdes toutes les opinions et toutes les conduites, mais il nous Ote par là le moyen d'en rejeter aucune comme fausse. Détruisant la vérité dogmatique, partielle ou abstraite, il insinue l'idée d'une verité totale..." ( $S$, 250). Après sa critique très détaillee du marxisme et de l'U.R.S.S., il semble que les questions qu'il a posées ne peuvent jamais être aussi ouvertes qu'autrefois. Car les questions parfois n'exigent pas de réponses. Peu à peu Merleau-Ponty est en train de reconnaître que les problèmes qu'il a releves trouvent leur solution dans une description claire de l'homme. Peut-être c'est l'homme lui-même qui est le problème. Il faut, peut-être, étudier l'homme dans tous ses aspects--language, art, politique-clarifier son rapport aux autres, surtout si ce rapport n'a pas d'appui comme l'histoire ou la religion sur lequel il se repose (SNS, 331). L'ambiguïté de l'existence révèle que le noyau humain c'est l'homme luimême. Marx l'a dit et c'est l'essentiel de la philosophie existentialiste. L'important est de continuer sa recherche sur l'homme. Enfin, l'homme demeurerait au monde sans idéologie ou dogme. Le "mystère humain" est de vivre sans autre reference que l'homme. L'histoire, selon MerleauPonty, est faite de violence, de cette cruauté que l'homme a envers autrui au nom de quelque croyance. Si le marxisme n'a pas le pouvoir de donner une solution à la violence humaine, ni à l'aliénation de l'homme dans

12"Complexité objective," 5.

13"Complexite objective", 9. Voir aussi "Indochine S.O.S." Les Temps modernes 18 (mars 1947), surtout les pages qui ne sont pas inclues dans Signes, pour le role qu'assume la France. 
le monde, ce n'est pas à cause de la vérité de la théorie mais de l'échec à l'appliquer. Toutes les grandes croyances de l'histoire aboutissent a la contingence humaine. Le problème qui reste est celui de l'intersubjectivite, de l'éthique, et demeure dans le cadre de l'existentialisme: comment vivre ensemble dans un monde fini sans recours à un Autre transcendant?

PATRICK J. PRYOR

\section{OUVRAGES CITES}

Arendt, Hannah. On Violence. New York: Harcourt, Brace, Jovanovich, 1970.

Burnier, Michel-Antoine. Les Existentialistes et la politique. Paris: Gallimard, 1966.

Meyer, Rudolf W. "Maurice Merleau-Ponty und das Schicksal des Franzosischen Existentialismus." Philosophische Rundschau 5 Helf 3/4 (1955): 129-165.

Hegel, G. W. F. La Phénoménologie de Pesprit. Traduction de Jean Hyppolite. Paris: Aubier, Editions Montaigne, Tome I, 1939.

Science de la logique. Premier tome. Premier livre. "l'Etre." [Edition de 1812]. Traduction, présentation et notes par PierreJean Labarrière et Gwendoline Jarczyk. Paris: Aubier Montaigne, 1972.

Merleau-Ponty, Maurice. "Complicité objective." Les Temps modernes 34 (juillet 1948): 1-11. (CO)

-...-.-. Humanisme et terreur. Essai sur le probleme communiste. Paris: Gallimard 1947. (HT)

-.....- "Indochine S.O.S." Les Temps modernes 18 (mars 1947):10391052.

......-. La Phénoménologie de la perception. Paris: Gallimard, 1945. (PP).

Sens et non-sens. Gème edition. Paris: Editions Nagel, 1966. (SNS).

Signes. Paris: Gallimard, 1960. (S).

Sève, Lucien. Marxisme et théorie de la personnalité. 2ème édition. Paris: Editions sociales, 1972. 